\title{
The Design of Smart Pipe Data Concentrator
}

\author{
Yulong Zhen \\ Beijing institute of Radio Metrology and Measurements \\ Beijing, China \\ zhenyulong1109@163.com
}

\begin{abstract}
In this paper, a design method of data centralized equipment for smart pipe is presented. Data Concentrator is responsible for collecting data of different interface sensors, and uploading those data to the central server via the Internet. Data concentrator sets up a LAN with terminal devices and sensing equipment, complete the data of terminal devices and sensing equipment collecting.
\end{abstract}

Keywords-SmartPipe; Dataconcentrator; ZigBee; WiFi; 3G; SimpliciTI

\section{INTRODUCTION}

In the construction of smart pipe ,Various sensing technology has been widely used, deployed multiple types of sensors. Each sensor is a source of information, collected environmental data in a certain frequency , and send data to the central server through $3 \mathrm{G}$ network, With the development of the smart pipe, the terminal equipment and sensor devices showing explosive growth trend, if each terminal equipment and sensing devices are provided by mobile operators to achieve communication with the central server, then the operating cost of the smart pipe is also an amazing number. In order to save operating costs, and make terminal devices and sensing devices communication with central server, it is necessary to develop such a device, it can be collected the data of sensors which has different interface, and send the data to central server through $3 G$ wireless network, all sensors share a $3 G$ channel, so the operating costs greatly reduced, we are calling it data concentrator.

Data concentrator set up local area network with terminal devices and sensing devices by a limited range wireless network, it supports the Zigbee protocol,433MHz short-range wireless communication and RS232, RS485 protocol cable support for up to 20 terminal devices and sensing device node. Data concentrator communicate with central server through 3G network, greatly reduces the cost of operating, support inquire the various configurations and equipment status through text messages, flexible and convenient.

\section{THE HARDWARE DESIGN}

In this paper, a new method is proposed to collect data from sensor and upload it to the server through 3G network. The data concentrator hardware circuit structure, the overall hardware circuit structure is shown in Figure 1.

\author{
Changwen Liu \\ Beijing institute of Radio Metrology and Measurements \\ Beijing, China
}

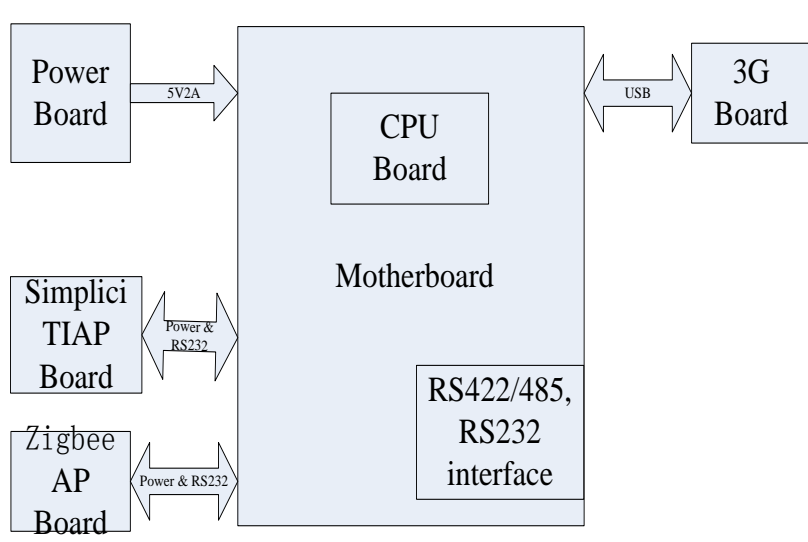

Figure 1 Hardware Circuit Structure of Data oncentrator

Data concentrator used modular thought in hardware design, which is based on a motherboard, each function module using hard-wired way connected to the motherboard. The hardware module is composed of the main board, power board, AP SimpliciTI board, AP Zigbee board, 3G module, WiFi module. The power board supply overall power, access to the motherboard through the power interface, the motherboard supply power to the other modules, and communication with other module through RS232 or USB protocol. The CPU board completed running the Linux operating system and the data concentrator software, complete communication with each module. AP SimpliciTI module communication with motherboard through RS232,obtain power from the motherboard, support SimpliciTI protocol,and achieve the AP function of SimpliciTI protocol.AP Zigbee module communication with motherboard through RS232,obtain power from the motherboard, support Zigbee protocol, and achieve the AP function of Zigbee protocol.3G module communicate with motherboard through USB, obtain power from the motherboard, support WCDMA protocol, and achieve the $3 \mathrm{G}$ function.

\section{THE SOFTWARE DESIGN}

Before you begin to format your paper, first write and save the content as a separate text file. Keep your text and graphic files separate until after the text has been formatted and styled. Do not use hard tabs, and limit use of hard returns to only one return at the end of a paragraph. Do not add any kind of pagination anywhere in the paper. Do not number text headsthe template will do that for you.

Data concentrator software is composed of the device driver, network protocol stack, standard function interface, 
equipment data terminal response services, data storage and transmission, server-side data service. Device drivers include network device driver, USB device driver and RS232 serial port device driver, network device driver support net equipment, USB device driver supports 3G and WiFi devices, RS232 supports ZigBee and 433 devices. The interface layer of the network protocol stack and the standard function is composed of the TCP/IP protocol stack and the standard AP RS232 function, which supports TCP protocol transmission and RS232 protocol standard. The device data response service is responsible for the transmission request, data transmission and storage of the data for each interface device. The server-side data service to complete the data uploaded to the central server.

Small and low power RF networks typically contain battery powered devices, which require a longer battery life. The SimpliciTI network protocol is a proprietary low power RF protocol for simple compact RF networks[1]. The SimpliciTI network protocol can simplify the implementation of the work, and reduce the resource occupancy of the microcontroller as much as possible[2].

SimpliciTI network protocol supports two basic network topologies, one is star network topology, and the other is peer to peer network[3][4]. Clusters of network structure is an extension of the star network topology and in clusters of network structure to achieve the communication of arbitrary node and data center (AP) node[5], as shown in Figure 2

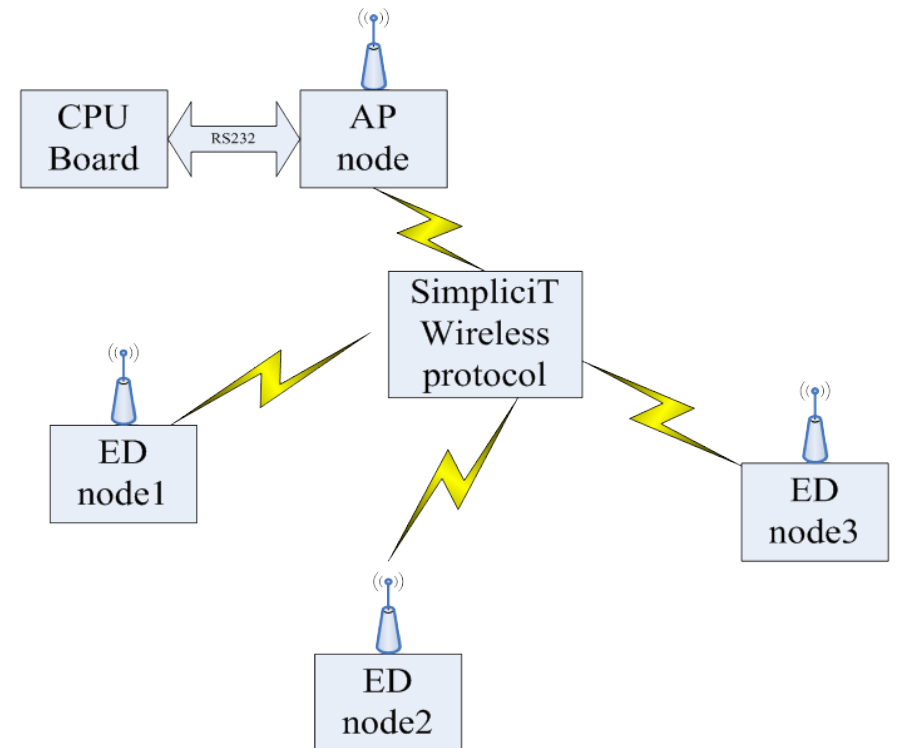

Figure 2 SimpliciTI protocol network structure diagram
ZigBee protocol network structure diagram is shown in Figure 3, ZigBeeAP board communication with motherboard through the RS232 interface, ZigBeeAP module to achieve the ZigBee protocol AP function, to achieve the terminal equipment management. ZigBee works in the $2.4 \mathrm{GHz}$ band, the transmission rate is $20 \mathrm{kbps} \sim 250 \mathrm{kbps}$, the transmission distance is $10 \mathrm{~m} \sim 100 \mathrm{~m}$.

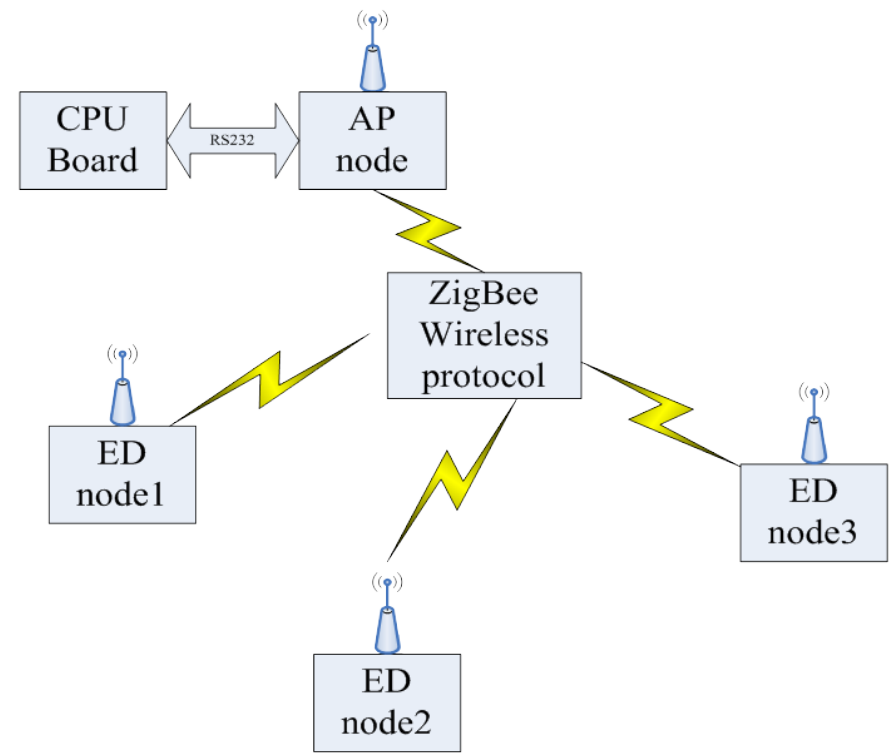

Figure 3 ZigBee protocol network structure diagram

\section{CONCLUSION}

The design-method of data concentrator can realize the sensor data collection in smart pipe, the sensing device and server data transmission more smoothly and greatly saving operation cost of the smart pipe.

\section{References}

[1] Friedman L. SimpliciTI developers notes [EB/OL].(2008-81)http://focus.ti.com.cn/cn/docs/toolsw /folders/print/simpliciti. html? DCMP = hpa _rf_general\&HQS=Other+OT+simpliciti.

[2] SimpliciTI-compatible UARTDriver[EB/OL].(2009-09-22) [2011-0827].http://focus.ti.com/lit/an/swra306/swra306.pdf.

[3] Introduction to SimpliciTI [EB/OL].(2008-12-01) [2011-0827].http://focus.ti.com.cn/cn/lit/ml/swru130b/swru130b.pdf

[4] CC1110 datasheet.Texas Instruments[EB/OL].http://www.ti.com

[5] TL. $<<$ A true system-on-chip solution for $2.4 \mathrm{GHz}$ IEEE 802.15.4/ZigBee >> [EB/OLJ.2010-1-20.http://focus.ti.com/cc2430.pdf] 